\title{
An Efficient Eigenvector Approach for Finding Netlist Partitions
}

\author{
Scott W. Hadley, Brian L. Mark, and Anthony Vannelli
}

\begin{abstract}
A fast eigenvector technique for obtaining good initial node partitions of netlists for use in interchange heuristics is described. The method is based on approximating the netlist or hypergraph by a weighted graph, $G$, such that the sum of the cut edges in $G$ tightly underestimates the number of cut nets in any netlist partition. An eigenvector technique of Barnes [2] is used to partition the graph $G$ into $k$ blocks of fixed module size. Another feature of this graph underestimation model of the netlist is that it allows us to obtain lower bounds on the actual number of cut nets. $A$ multiblock node interchange heuristic of Sanchis [20] is tested on the one resulting netlist partition obtained by this new eigenvector approach on a variety of small to large sized benchmark netlist partitioning problems (between 300 to 12000 modules and nets). Test results on the larger netlists show that in most cases this eigenvector-node interchange approach yields netlist partitions with comparable or fewer cut nets than the best netlist partitions obtained by using node interchange heuristics alone on many random initial netlist partitions. Moreover, the running time of this method is a small fraction of the previous node interchange methods.
\end{abstract}

\section{INTRODUCTION}

$\mathrm{T}$ HE partitioning of the modules of a netlist arises in many areas, including the laying out of circuits on computer chips and printed circuit boards [4], [11], [19], computer program segmentation [5], [8], [10], and the laying out of machines in advanced manufacturing systems [22], [23]. In each case, we assume that the netlist can be represented by a hypergraph, which is a generalization of a graph where a hypergraph generalized edge (net) can connect more than two modules. Throughout our discussion, we assume that the netlist or hypergraph $H$, contains $n$ nodes (modules) $V=\{1,2, \cdots, n\}$ and $|E|$ generalized edges (nets). We study the problem of partitioning the $n$ nodes into $k$ disjoint blocks, $V_{1}, V_{2}, \cdots$,

Manuscript received January 7, 1991; revised June 16, 1991. This work was supported by an operating grant (OGP 0044456) and a Microelectronics Research Fund grant from the Natural Sciences and Engineering Research Council of Canada. This paper was recommended by Associate Editor M. Marek-Sadowska.

S. W. Hadley was with the Department of Electrical and Computer Engineering, University of Waterloo, Waterloo, Ont., Canada. He is now with the Department of Mathematics and Systems Engineering, Shell Research B.V., Amsterdam, The Netherlands.

B. L. Mark was with the Department of Electrical and Computer Engineering, University of Waterloo, Waterloo, Ont., Canada. He is now with the Department of Electrical Engineering, Princeton University, Princeton, NJ 08540 .

A. Vannelli is with the Department of Electrical and Computer Engineering, University of Waterloo, Waterloo, Ont., Canada N2L 3G1.

IEEE Log Number 9107118
$V_{k}$, of node sizes $m_{1}, m_{2}, \cdots, m_{k}$, respectively, such that the number of hypergraph edges connecting the $k$ blocks is minimized.

Since this problem is known to be NP-complete (even in the simpler case where the hypergraph $H$ is a graph $G$ ([12]), heuristics have been developed to obtain suitable partitions [10], [16], [20]. These heuristics are known as interchange methods and are based on iteratively improving a series of partitions. In general, the quality of the final solution depends on the quality of the initial (starting) partition. In the heuristics described above the initial partition is randomly generated.

In this paper, we present a fast method for obtaining an initial partition to which any interchange method can be applied. The method is based on approximating the netlist or hypergraph by a weighted graph, $G$, that tightly underestimates the number of cut nets in any netlist partition. An eigenvector technique of Barnes [2] is used to partition the resulting graph, $G$, into $k$ blocks of fixed module size. A novel feature of this graph underestimation model of the netlist is that it allows us to obtain lower bounds on the actual number of cut nets. An efficient implementation of the Sanchis node interchange heuristic is developed to further reduce the number of nets connecting $k$ blocks [20].

This node interchange heuristics is tested on the one resulting netlist partitioned obtained by this new eigenvector approach on a variety of small to large sized benchmark netlist partitioning problems (between 300 to 12000 modules and nets). Test results on the larger netlists indicate that in most cases this eigenvector-node interchange approach yields netlist partitions with comparable or fewer cut nets than the best netlist partitions obtained by using node interchange heuristics alone on many random initial netlist partitions. Also, the running time of this method is a small fraction of the previous node interchange methods.

The advantage of developing an eigenvector approach to solve the partitioning problem is that the generalized initial partitions tend to have many nodes placed in the "right blocks." This is due to the observation that eigenvector methods are more global for solving large-scale optimization problems. For example, eigenvector approaches have been used to solve many VLSI placement problems [3], [14].

On the other hand, node interchange methods are greedy or local in nature and get easily trapped in local optima. 
More important, it has been shown that interchange methods may not converge to "optimal" or "near optimal" partitions unless they initially begin from "good" partitions [13], [17]. We use an eigenvector approach to attempt to place most of the nodes in the correct blocks or node partitions for large-scale partitioning problems. An efficient node interchange approach is developed to move nodes that reduce the number of generalized hypergraph edges that connect modules in at least two blocks.

This paper is divided into five sections. Section II discusses how a netlist $H$ can be estimated by a weighted graph, $G$. The edge weights of the graph $G$ are determined by characterizing the solution of a related $\ell_{p}(p \geq 1)$ minimization problem. In Section III, we discuss how the graph $G$ can be used to obtain initial node partitions using the eigenvector based approach of Barnes [2]. Lower bounds on the number of cut nets are obtained using the tightly underestimated graph approximation, $G$, of $H$ and the simple eigenanalysis results from Donath and Hoffman [9]. The testing of this new eigenvector-based model and the efficient multiblock interchange method of Sanchis [20] is conducted on a variety of well-known benchmark test problems from VLSI circuit layout design and is presented in Section IV. Conclusions and directions for future research are given in Section $\mathrm{V}$.

\section{Approximating a Netlist by a Graph}

The netlist partitioning problem can easily be described as a hypergraph partitioning problem. In general, hypergraph partitioning problems are NP-hard, even in the simple case where the hypergraph is a graph [12]. However, there are cases where good heuristics exist for partitioning problems on graphs but not for the corresponding hypergraph partitioning problem. The problem of partitioning the modules of a netlist into blocks of specified size such that the number of nets with modules in more than one block is minimized falls into this category. In this case a good heuristic based on the eigenvalues and eigenvectors of the adjacency matrix of the graph has been given by Barnes [2]. As such, we are interested in finding ways to approximate a hypergraph by a graph. A method for approximating a netlist $H$ by a graph, $G$, with weighted edges is given in [25], where we consider partitioning the netlist into two blocks only with no block size constraint. The node set of $G$ is the same as the node set of $H$. The edge set of $G$ is obtained by replacing each net of $H$ by the edge set of a clique containing the modules of the net. Edge weights are assigned so that $G$ provides an underestimation of $H$. We say that $G$ underestimates $H$ if the weight of the edges of $G$ that are $c u t$ by any module partition is not greater than the number of nets cut by the same partition. An underestimation can prove to be useful when either a lower bound or the optimal solution for the graph partitioning problem can be found. The reason the underestimation is useful is that any lower bound for the graph partitioning problem will also provide a lower bound for the netlist partitioning problem. If a general estimation is used, bounding results from graph partitioning cannot be exploited.

In this section, we extend the technique described in [25] to obtain weights for the edges of $G$ when we consider partitioning the netlist into $k$ blocks of specified sizes. In subsection $\mathrm{A}$, we find a characterization of the weights on the approximating graph of the netlist. We provide an illustrative example using a netlist with five modules and three nets at the end of the section.

\section{A. Determining Edge Weights}

We generate the edge weights by considering the clique obtained by each generalized edge in turn. After considering each generalized edge we obtain a graph containing multiple edges. (If node $i$ and node $j$ are contained in $t$ generalized edges, there will be $t$ (multiple) edges between $i$ and $j$ in the new graph.) We generate $G$ by replacing each set of multiple edges by a single edge whose weight is the sum of the weights of the multiple edges.

By focusing on a particular generalized edge (net), we wish to assign values such that the weight of any cut in the clique underestimates any cut in the generalized edge. We show that all edge weights in the corresponding clique will have the same weight when we consider a single generalized edge (see Theorem 1).

Assume for simplicity that each generalized edge (net) has a unit weight and that we wish to partition the nodes (modules) among $k$ blocks. Let $a$ be the value assigned to each edge of the clique representation of a generalized edge. In order to find a graph fit that underestimates a partition of the netlist, we must have $|\varphi(\ell)| a \leq 1$, where $|\varphi(\ell)|$ is the number of cut edges in the $\ell$ th distinguishable partition of the net into $k$ blocks. There are only a finite number of distinguishable partitions of the modules of a net. In order to minimize the error in the underestimation of a cut of generalized edge, we must choose the maximum value for $a$. The maximum possible value for $a$ is

$$
\frac{1}{\max _{\ell}(|\Sigma(\ell)|} \text {. }
$$

For example, in the case of a net connected to four modules where these modules are partitioned over two blocks, we see that the maximum number of interconnections arises if we assign two modules in each block. This implies that there are four interconnections between the two blocks. Thus $a=1 / 4$. The best four-node (modules) approximation of the netlist where the four modules are connected to one net is shown in Fig. 1.

\section{B. Theoretical Derivation of Optimal Edge Weights}

In this subsection, we expand on the above observations by giving a proof that the intuitive edge weighting (1) is in fact optimal in the underestimation case. In general, to edge $(i, j)$ in $G$ we assign weight $a_{i j}$. Given a partition of the nodes, we want the edge weights to satisfy the property that, if the generalized edge is cut by a par- 


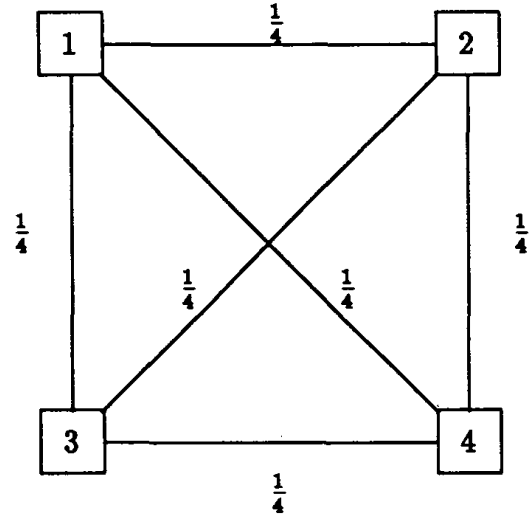

tition, then the sum of the edge weights of the cut edges in $G^{\prime}$ is less than or equal to 1 (i.e., the value underestimates the number of cut edges in the hypergraph).

We employ the following notations and definitions when finding the best underestimation edge weighting for these graphs.

Definition 1: A $k$-partition of the nodes $V$ of the complete graph $K_{n}$ is a partition of $V=\left\{V_{1}, V_{2}, \cdots, V_{k}\right\}$ such that $V_{i} \cap V_{j}=\emptyset \forall i \neq j$, and $\cup_{i=1}^{k} V_{i}=V$. Let $P$ be the set of all $k$-partitions of the nodes of a graph.

Definition 2: An equal partition of the node set $V$ is a $k$-partition such that ||$V_{i}|-| V_{j}|| \leq 1 \forall i, j$. We let $\mathcal{E}$ be the set of all equal partitions of the node set of a graph.

We can now give a system of equations describing the underestimation property described above:

$$
\begin{aligned}
\sum_{(i, j) \in \varphi(\ell)} a_{i j}+s_{\ell} & =1 \\
s_{\ell} & \geq 0
\end{aligned}
$$

Fig. 1. Best four-module graph approximation.

easy to verify that the number of edges cut by $\ell \in P$ is

$$
|\varphi(\ell)|=\left(\begin{array}{l}
n \\
2
\end{array}\right)-\sum_{i=1}^{k}\left(\begin{array}{c}
\left|V_{i}\right| \\
2
\end{array}\right)
$$

Without loss of generality, assume $\left|V_{i}\right|=\left|V_{2}\right|+t$ for some $t \geq 2$, (i.e., $\ell \notin \mathcal{E}$ ). Consider the $k$-partition $\hat{\ell}=$ $\left\{\hat{V}_{1}, \hat{V}_{2}, \cdots, \hat{V}_{k}\right\}$, where $\hat{V}_{1}$ and $\hat{V}_{2}$ are obtained by taking one node from $V_{1}$ and placing it in $V_{2}$, giving $V_{1}$ and $\hat{V}_{2}$, respectively, with $\hat{V}_{i}=V_{i}$ for $i \geq 3$. By expansion and comparison it follows that

$$
\begin{aligned}
|\varphi(\hat{\ell})| & =|\varphi(\ell)|-(1-t) \\
& >|\varphi(\ell)| .
\end{aligned}
$$

This is a contradiction.

Lemma 2: Assume there exists an optimal solution to $S_{p}, p \geq 1$ with $a_{i j}=a$ for all $i, j$; then there exists an optimal solution where

$$
a_{i j}^{\mathrm{opt}}=a^{\mathrm{opt}}=|\varphi(\ell)|^{-1}
$$

where $\ell \in \mathcal{E}$.

Proof: Given any feasible solution where $a_{i j}=a$ $\forall i, j$, we begin by noticing that for all $\ell \in P, s_{\ell}$ decreases as $a$ increases. Therefore, we are interested in finding the largest value of $a$ (implying smallest error for each partition) that yields a feasible solution. Since there exists a feasible solution with $a=0$, it follows that $a^{\text {opt }} \geq 0$.

Since

$$
s_{\ell} \geq 0, a^{\text {opt }} \geq 0, \text { and }|\varphi(\ell)| \geq 0,
$$

it follows that

$$
a^{\text {opt }} \leq|\varphi(\ell)|^{-1}, \forall l \in P .
$$

So, the largest value of $a$ occurs when $|\varphi(\ell)|^{-1}$ is minimized over all $\ell \in P$, i.e., $|\varphi(\ell)|$ is maximized. From Lemma 1 , we know that this occurs when $l$ is an equal $k$-partition. Thus, $a^{\text {opt }}=|\varphi(\ell)|^{-1}$ for $\ell \in \mathcal{E}$.

Lemma 3: For $\ell \in \mathcal{E}$,

$$
|\varphi(\ell)|=\left(\begin{array}{l}
n \\
k
\end{array}\right)-\left(\begin{array}{c}
\left\lfloor\frac{n}{k}\right\rfloor \\
2
\end{array}\right)\left(k\left(1+\left\lfloor\frac{n}{k}\right\rfloor\right)-n\right)\left(\begin{array}{c}
{\left[\begin{array}{l}
n \\
k
\end{array}\right\rfloor} \\
2
\end{array}\right)\left(n-k\left\lfloor\frac{n}{k}\right\rfloor\right) .
$$

Proof: Since $\ell \in \mathcal{E}$, there are $(n-k\lfloor n / k\rfloor)$ subsets for all $\ell \in P$. We can now formulate the problem of finding a best (underestimation) edge weighting a mathematical programming problem. There are various measures for determining a best edge weighting. Likely candidates are the $l_{p}$ norms for $p=1,2, \infty$. We let $S_{p}$ denote the mathematical programming problem

$$
S_{p}=\min \left\{l_{p}(a, s) \mid(a, s) \text { satisfies (2), (3) }\right\},
$$

where $l_{p}(a, s)$ is the $l_{p}$ norm of the elements of $s$.

Lemma 1: If $\ell=\left\{V_{1}, V_{2}, \cdots, V_{k}\right\}$ is a $k$-partition of the nodes of the complete graph $K_{n}$ that maximizes the number of edges in $\varphi(\ell)$ over all $k$-partitions, then $\ell$ is an equal partition (i.e., $\ell \in \mathcal{E}$ ).

Proof: We show this result by contradiction. It is of size $\lceil n / k\rceil$ and the remaining $k(1+\lfloor n / k\rfloor)-n$ subsets are of size $\lfloor n / k\rfloor$. The number of edges in a subset of size $t$ is $(t / 2)$. The result follows.

Theorem 1: There exists an optimal solution to $S_{p}$, $p \geq 1$ with

$$
a_{i j}^{\mathrm{opt}}=|\varphi(\ell)|^{-1} \forall i, j
$$

for any $\ell \in \mathcal{E}$.

Proof: First we define equivalence classes of all feasible partitions. Let $F_{d}$ denote the equivalence class of partitions that yields the same partition after permuting the graph nodes. Now, let $a^{*}=\left(a_{i j}^{*}\right)$ denote an optimal edge weighting, let $s^{*}=\left(s_{\ell}^{*}\right)$ be the corresponding errors, and let $\mathrm{err}_{d}$ denote the sum of the errors for all partitions 
TABLE I

Calculation of $a$ FOR DifFERENT Numbers OF $\operatorname{BLOCKS}(k)$

\begin{tabular}{cccc}
\hline$n$ & $k=2$ & $k=3$ & $k=4$ \\
\hline 2 & 1 & 1 & 1 \\
3 & $1 / 2$ & $1 / 3$ & $1 / 3$ \\
4 & $1 / 4$ & $1 / 5$ & $1 / 6$ \\
5 & $1 / 6$ & $1 / 8$ & $1 / 9$ \\
6 & $1 / 9$ & $1 / 12$ & $1 / 13$ \\
7 & $1 / 12$ & $1 / 16$ & $1 / 18$ \\
\hline
\end{tabular}

\section{Net \#1}

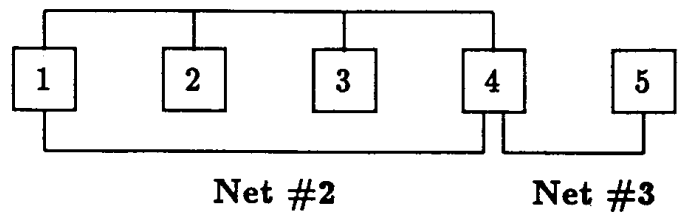

Fig. 2. Five-module, three-net example.

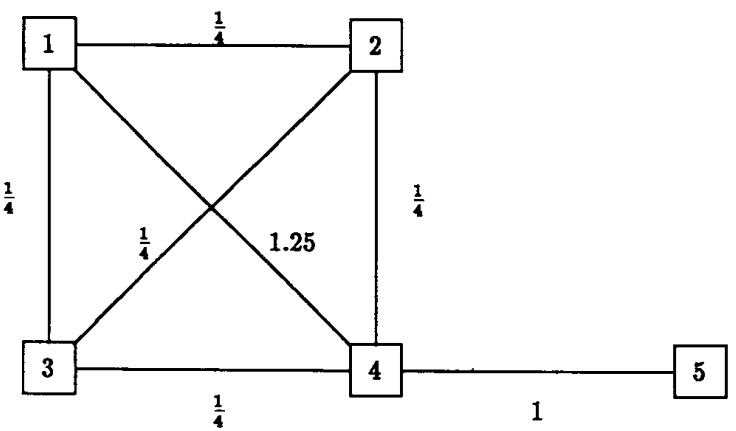

Fig. 3. Best four-module graph approximation.

in equivalence class $d$; i.e.,

$$
\mathrm{err}_{d}=\sum_{\mathcal{P} \in F_{d}} s_{\ell}^{*}
$$

Since the $\ell_{p}$ norm ( $p \geq 1$ and finite) of the errors is convex in the feasible region (i.e., underestimations), it is easy to see that the minimum of the errors subject to (6) for all $d$ occurs when all errors $s_{p}^{*}$ are equal for each equivalence class $d$. By symmetry of the complete graph it follows that this equality holds when all edge weights are equal. It follows that there exists an optimal solution with all edge weights equal. Applying Lemma 2 the theorem follows for finite $p$. For the $\ell_{\infty}$ case a similar argument based on symmetry can be used.

Table I shows the calculation of the values $a_{i j}^{\text {opt }}$ (using Theorem 1 and Lemma 3) for different modules and required blocks.

We now illustrate this technique by means of a small five-module, three-net example and obtain the best graph underestimation. The hypergraph corresponding to the netlist is shown in Fig. 2, and the best graph underestimation is shown in Fig. 3 .

Each edge in the clique on modules $\{1,2,3,4\}$ will get weights of $1 / 4$, the edges of cliques $\{1,4\}$ and $\{4,5\}$ will get weights of 1 . Notice that modules 1 and 4 occur in two nets together: Net \#1 with four modules and Net \#2 with two modules. Therefore the weight of edge $(1,4)$ in $G$ has weight $1.25=1+1 / 4$.

\section{Generating Initial Partitions and Bounds}

Given the graph approximation, $G$, of the hypergraph $H$, we find an initial partition of graph $G$ by using the eigenvector-based approach of Barnes [2]. We will use the resulting partition of the weighted graph $G$ as a partition for the hypergraph (netlist) $H$. Barnes shows that the graph partitioning problem is equivalent to a matrix approximation problem. We summarize these results below.

Assume that the approximating graph, $G$, under consideration has $n$ nodes that are to be partitioned into $k$ disjoint blocks of sizes $m_{1} \geq m_{2} \geq \cdots \geq m_{k}$. A partition can be completely specified by a set of $k$ node assignment vectors, $x_{1}, x_{2}, \cdots, x_{k}$, one corresponding to each block, which have the form

$$
\boldsymbol{x}_{j}=\left(x_{1 j}, x_{2 j}, \cdots, x_{n j}\right)^{T}, \quad 1 \leq j \leq k
$$

where

$$
x_{i j}= \begin{cases}1 & \text { if node } i \text { is in block } j \\ 0 & \text { otherwise. }\end{cases}
$$

Let $v_{i j}$ be the $i$ th component of the eigenvector corresponding to the $j$ th largest eigenvalue of the adjacency matrix of $G$. Barnes [2] shows that the solution of the following linear transportation problem gives an approximate solution to the graph partitioning problem:

$$
\operatorname{Maximize} \sum_{i=1}^{n} \sum_{j=1}^{k} \frac{v_{i j}}{\sqrt{m_{j}}} x_{i j}
$$

subject to $\sum_{i=1}^{n} x_{i j}=m_{j}, j=1, \cdots, k$

$$
\begin{aligned}
\sum_{j=1}^{k} x_{i j} & =1, \quad i=1, \cdots, n \\
x_{i j} & \geq 0, \quad i=1, \cdots, n ; j=1, \cdots, k .
\end{aligned}
$$

Technically, there are $2^{k}$ transportation problems (7) that should be solved where $k$ is small. Barnes [2] describes a selection procedure that allows one transportation problem to be solved. A powerful interior point algorithm proposed by Adler et al. is used to solve the linear transportation problem (7) [1].

In the two-block case, the transportation problem (7) can be further simplified by replacing $x_{i 2}$ by $1-x_{i 1}$. Making this substitution and letting $x_{i}=x_{i 1}$, the objective function becomes

$$
\sum_{i=1}^{n}\left\{\frac{v_{i 1}}{\sqrt{m_{1}}}-\frac{v_{i 2}}{\sqrt{m_{2}}}\right\} x_{i}+\sum_{i=1}^{n} \frac{v_{i 2}}{\sqrt{m_{2}}} .
$$


The transportation problem (7) reduces to the following $\{0,1\}$-knapsack problem:

$\operatorname{Maximize} \sum_{i=1}^{n}\left\{\frac{v_{i 1}}{\sqrt{m_{1}}}-\frac{v_{i 2}}{\sqrt{m_{2}}}\right\} x_{i}=\sum_{i=1}^{n} \delta_{i} x_{i}$

subject to $\sum_{i=1}^{n} x_{i}=m_{1}, \quad x_{i} \in\{0,1\}, \quad i=1, \cdots, n$.

It is well known that the solution to (9) is obtained by sorting the objective coefficients in nonincreasing order and setting $x_{i}=1$ for the first $m_{1}$ variables in the sorted list (all other variables are set to zero).

Recall the weight of any cut in the generated graph $G$ underestimates the number of generalized edges cut in $H$. Donath and Hoffman [9] introduced an approach that finds lower bounds on the weight of any cut of $G$. Thus we can find a lower bound on the number of cut generalized edges of $H$.

Consider the matrix $\boldsymbol{A}=\left[a_{i j}\right]$, where $a_{i j}$ is the weight of the edge joining nodes $i$ and $j$ (i.e., $A$ is the adjacency matrix of $G$ ). The matrix $A$ is symmetric and of order $n$ with zeros along the main diagonal. Consider any diagonal matrix $\boldsymbol{U}=\left[u_{i j}\right]$, where

$$
\sum_{i=1}^{n} u_{i i}=-\sum_{i} \sum_{j} a_{i j} \text {. }
$$

Then the main result of Donath and Hoffman [9] is

$$
E_{c} \geq-\frac{1}{2} \sum_{i=1}^{k} m_{i} \lambda_{i}(\boldsymbol{A}+\boldsymbol{U}),
$$

where $E_{c}$ is the sum of the edges cut by the optimal partition and $\lambda_{i}$ is the $i$ th largest eigenvalue of $\boldsymbol{A}+\boldsymbol{U}$.

Now, let $E_{H}$ be defined as the sum of the nets cut by the optimal partition. Since the number of cut edges for any partition of the generated graph $G$ underestimates the number of cut nets for the same partition in the netlist, it follows that the sum of the optimal cut edges, $E_{c}$, is less than the sum of the optimal cut nets, $E_{H}$; that is,

$$
E_{H} \geq E_{c} \geq-\frac{1}{2} \sum_{i=1}^{k} m_{i} \lambda_{i}(A+U) .
$$

The bound provided by inequality (12) shows the importance of tightly underestimating the number of cut nets by the cut edges of the graph $G$ given in subsection II-B.

We now illustrate the concepts that have been introduced by finding the best weighted graph approximation, partition, and lower bound on the smallest number of nets that are cut on the simple five-node, three-net example given in Fig. 2. The best underestimation graph fit for this netlist is given in Fig. 3.

Assume that we wish to partition the netlist into two blocks, one block containing three nodes and the other block two. The largest two eigenvalues of the adjacency matrix $\boldsymbol{A}\left(a_{i j}\right.$ containing the weighted values connecting nodes $i$ and $j$ ) of this graph and the corresponding eigen- vectors are

$$
\begin{aligned}
& \lambda_{1}=1.7368, \quad v_{1}=[0.548,0.206,0.206,0.679,0.391], \\
& \lambda_{2}=0.277, v_{2}=[0.223,0.535,0.535,-0.164,-0.592] .
\end{aligned}
$$

Substituting into (8), we find that the coefficients of the objective function are

$$
\delta=[0.158,-0.259,-0.259,0.508,0.645] .
$$

The partition obtained by sorting the components in $\delta$ is

$$
\begin{aligned}
& S_{1}=\{1,4,5\} \\
& S_{2}=\{2,3\} .
\end{aligned}
$$

Donath and Hoffman [9] obtain bounds on the number of cut edges in a graph by choosing the diagonal elements of $\boldsymbol{A}+\boldsymbol{U}$ by setting

$$
\begin{aligned}
& u_{i i}=-\sum_{j} a_{i j} \\
& u_{i j}=0 \quad \text { for } i \neq j .
\end{aligned}
$$

In other words, the diagonal elements, $u_{i i}+\boldsymbol{A}+\boldsymbol{U}$ form the negative sum of all the weighted edges connected to node $i$.

We find that

$$
E_{H} \geq E_{c} \geq 0.719
$$

This implies that at least one (i.e., $\lceil 0.719\rceil$ ) net is cut. Since the partition generated by $S_{1}$ and $S_{2}$ cuts exactly one net, we have provably obtained the optimal partition.

\section{Test Results}

An available FORTRAN/C code, NETPART, has been developed on a UNIX environment to incorporate the eigenvector-based model partitioning method desribed in the preceding two sections. We present experimental results from applying the eigenvector method and the node interchange method to several networks. All computational work was done on a MIPS/2000 RISC computer at the University of Waterloo.

The FORTRAN subroutine LASO [21] was used to obtain the $k$ largest eigenvalues and their corresponding eigenvectors. The LASO code is based on the block Lanczos method for finding a few largest or smallest eigenvalues and corresponding eigenvectors of a sparse matrix [7].

The calculation of the $k$ largest eigenvalues and corresponding eigenvectors for larger netlists by LASO is modest [21]. This is accomplished by deleting the nets connected to a large number of modules ( $>20$ modules). This has the effect of not introducing large-sized cliques in the graph with weighted edges; that is, the approximating graph of the netlist is kept sparse. These nets were put back into the netlist when the Sanchis multiblock interchange heuristic was used and the lower bounds were determined by inequality (12). 
In the two-block case, a simple heap sort is used to solve the transportation problem (9). A general transportation problem solver using an interior point method was developed to handle the multiple-block case [1]. Our results show that the time required to solve the transportation problem is not significant in comparison with the time required to obtain the eigenvalues and eigenvectors using the block Lanczos code LASO.

A C program implementation of the efficient multiblock node interchange approach of Sanchis [20] which further reduces the number of cut nets of any initial partition was developed. This node interchange heuristic is capable of handling partitions involving an arbitrary number of blocks, and includes the feature of level gains to distinguish between node moves. Our implementation allows for nonuniform net weights without significantly changing the time complexity of the Sanchis method.

These techniques and the resulting computer codes were tested on five netlist partitioning problems, listed in Table II. Chipl is taken from the work of Fiduccia and Mattheyses [10] and Primary1, Primary2, Bio, and Industry2 are taken from the MCNC gate-array and standard cell test suite benchmarks. These netlists vary in size from 300 to 12000 nodes and 300 to 13000 nets.

The preceding eigenvector-based procedure was tested on the netlists listed in Table II. In all cases, we attempted to partition the netlists into two, four, or six disconnected blocks of modules. Each block was allowed to have up to $10 \%$ more or less than the equipartitioned number of modules.

Table III shows the number of cut nets and running time to obtain these results by applying the eigenvector approach of Sections II and III to these netlists. The CPU time for the partition cuts include the time for forming the graph adjacency matrix, solving for the eigenvalues and eigenvectors, and finally solving the transportation problem.

Table IV shows the results obtained by supplying the multiblock node interchange heuristic of Sanchis [20] to improve the number of cut nets found using the eigenvector approach for the two, four, and six block cases given in Table III.

Tables V, VI, and VII give the results obtained from using node interchange on 30 random starting partitions. The level parameter setting was set to " 1 "' in all test cases [20, pp. 64-66]. It was found that level parameter settings up to 4 led to negligible partition improvement on these test cases. One should note that the average net size does not exceed 4 for any of the test problems. However, the running times were substantially larger using level parameter settings larger than 1 .

Each of these three tables contains seven columns. The second column gives the average number (mean) of cut nets obtained for each netlist example. Column 3 gives the standard deviation; columns 4 and 5 give the "best" and "worst" partitions obtained for each example. Finally, the last two columns yield the running time (second) and the number of runs that were conducted.
TABLE II

Netlist Partitioning Test Cases

\begin{tabular}{|c|c|c|c|c|c|c|}
\hline \multirow[b]{2}{*}{ Name } & \multirow[b]{2}{*}{ Nets } & \multirow[b]{2}{*}{ Nodes } & \multicolumn{2}{|c|}{ Node Degree } & \multicolumn{2}{|c|}{ Net Size } \\
\hline & & & $\bar{x}$ & $\sigma$ & $\bar{x}$ & $\sigma$ \\
\hline Chipl & 294 & 300 & 2.82 & 1.15 & 2.87 & 1.39 \\
\hline Primary 1 & 904 & 833 & 3.50 & 1.29 & 3.22 & 2.59 \\
\hline Primary 2 & 3029 & 3014 & 3.72 & 1.55 & 3.70 & 3.82 \\
\hline Bio & 5711 & 6417 & 3.26 & 1.03 & 3.66 & 20.92 \\
\hline Industry2 & 12949 & 12142 & 3.89 & 1.79 & 3.64 & 11.15 \\
\hline
\end{tabular}

TABLE III

EIGENVECTOR PARTITIONS

\begin{tabular}{|c|c|c|c|c|c|c|}
\hline \multirow[b]{2}{*}{ Name } & \multicolumn{2}{|c|}{2 Blocks } & \multicolumn{2}{|c|}{4 Blocks } & \multicolumn{2}{|c|}{6 Blocks } \\
\hline & Cuts & Time & Cuts & Time & Cuts & Time \\
\hline Chip 1 & 42 & 5.02 & 80 & 10.19 & 91 & 14.58 \\
\hline Primary 1 & 181 & 13.58 & 298 & 74.16 & 329 & 32.10 \\
\hline Primary 2 & 694 & 48.61 & 925 & 235.16 & 1097 & 238.69 \\
\hline Bio & 364 & 134.74 & 873 & 209.85 & 906 & 341.68 \\
\hline Industry2 & 1383 & 201.78 & 4472 & 292.20 & 4102 & 614.14 \\
\hline
\end{tabular}

TABLE IV

AFTER NODE INTERCHANGE

\begin{tabular}{|c|c|c|c|c|c|c|}
\hline \multirow[b]{2}{*}{ Name } & \multicolumn{2}{|c|}{2 Blocks } & \multicolumn{2}{|c|}{4 Blocks } & \multicolumn{2}{|c|}{6 Blocks } \\
\hline & Cuts & Time & Cuts & Time & Cuts & Time \\
\hline Chipl & 15 & 2.4 & 51 & 2.36 & 69 & 1.66 \\
\hline Primary 1 & 63 & 3.33 & 120 & 5.36 & 149 & 7.08 \\
\hline Primary 2 & 257 & 9.97 & 440 & 15.42 & 581 & 16.95 \\
\hline Bio & 175 & 18.67 & 262 & 17.87 & 369 & 36.04 \\
\hline Industry 2 & 530 & 39.32 & 1743 & 217.73 & 1689 & 190.10 \\
\hline
\end{tabular}

TABLE $\mathrm{V}$

Two-Block Partitions: Node Interchange

\begin{tabular}{lrrrrrr}
\hline \multicolumn{1}{c}{ Name } & \multicolumn{1}{c}{$\bar{x}$} & \multicolumn{1}{c}{$\sigma$} & Best & Worst & \multicolumn{1}{c}{ Time } & Runs \\
\hline Chip1 & 24.23 & 4.62 & 20 & 38 & 18.34 & 30 \\
Primary1 & 71.17 & 11.09 & 53 & 101 & 73.57 & 30 \\
Primary2 & 255.67 & 39.44 & 172 & 327 & 355.91 & 30 \\
Bio & 165.23 & 30.71 & 93 & 225 & 646.06 & 30 \\
Industry2 & 774.37 & 206.99 & 393 & 1176 & 1585.03 & 30 \\
\hline
\end{tabular}

TABLE VI

Four-Block Partitions: Node Interchange

\begin{tabular}{lrrrrrr}
\hline \multicolumn{1}{c}{ Name } & \multicolumn{1}{c}{$\bar{x}$} & \multicolumn{1}{c}{$\sigma$} & Best & Worst & \multicolumn{1}{c}{ Time } & Runs \\
\hline Chip1 & 63.53 & 7.20 & 53 & 79 & 37.66 & 30 \\
Primary1 & 180.93 & 15.35 & 140 & 204 & 137.55 & 30 \\
Primary2 & 784.63 & 43.39 & 625 & 845 & 887.41 & 30 \\
Bio & 645.80 & 46.96 & 578 & 704 & 719.21 & 30 \\
Industry2 & 2474.40 & 107.73 & 2266 & 2549 & 1365.18 & 30 \\
\hline
\end{tabular}

TABLE VII

SiX-Block Partition: Node InTERChange

\begin{tabular}{lrrrrrr}
\hline \multicolumn{1}{c}{ Name } & \multicolumn{1}{c}{$\bar{x}$} & \multicolumn{1}{c}{$\sigma$} & Best & Worst & \multicolumn{1}{c}{ Time } & Runs \\
\hline Chip1 & 83.60 & 7.31 & 69 & 97 & 52.20 & 30 \\
Primary1 & 215.94 & 13.42 & 187 & 240 & 199.65 & 30 \\
Primary2 & 913.23 & 34.39 & 806 & 961 & 2026.82 & 30 \\
Bio & 863.10 & 42.62 & 757 & 952 & 2951.34 & 30 \\
Industry2 & 2881.90 & 90.80 & 2513 & 3025 & 18114.67 & 30 \\
\hline
\end{tabular}


TABLE VIII

EIGENVECTOR BOUNDS

\begin{tabular}{|c|c|c|c|c|c|c|}
\hline \multirow[b]{2}{*}{ Name } & \multicolumn{2}{|c|}{2 Blocks } & \multicolumn{2}{|c|}{4 Blocks } & \multicolumn{2}{|c|}{6 Blocks } \\
\hline & Cuts & Time & Cuts & Time & Cuts & Time \\
\hline Chip1 & 7 & 12.27 & 19 & 22.56 & 28 & 36.45 \\
\hline Primary 1 & 21 & 28.71 & 49 & 40.71 & 56 & 71.26 \\
\hline Primary 2 & 93 & 80.16 & 191 & 194.39 & 224 & 386.74 \\
\hline Bio & 51 & 209.17 & 83 & 299.38 & 119 & 443.66 \\
\hline Industry2 & 187 & 297.63 & 515 & 421.74 & 614 & 803.07 \\
\hline
\end{tabular}

Some general observations can be made from the results presented in Tables II-VII. The final partitions that are obtained using the node interchange approach from the initial partition generated by the eigenvector technique compare favorably with those obtained using only random starting partitions. The total of the execution times for obtaining a starting partition using the eigenvector approach and then applying iterative improvement on this one generated partition is much less than the time required to perform the heuristic from 30 random starting partitions. For the four larger examples, the combined time of eigenvector and iterative improvement is from four to more than 20 times faster than using up to 30 random starting partitions.

In all cases, the results from the cascaded procedure of applying the eigenvector method and then iterative improvement are close to the best partitions that were obtained using iterative improvement alone from the random starting partitions. For most of the larger sized problems-Primary1, Primary2, Bio, and Industry2-this new approach yields cuts that are $50 \%-100 \%$ better than the cuts found for the partitions generated from random starting partitions only. Also, the quality of the resulting partitions is much better as the size of the problem increases and the number of blocks increases. The results are superior for the four and six block cases.

More important, the large test problems Bio and Industry 2 show the importance of getting the best partition possible from using a node interchange approach only once. The running time of the interchange method from one starting partition is becoming expensive. Thus, the method becomes more attractive for solving these practical large partitioning problems.

Table VIII shows lower bounds obtained on the number of cut nets for the five tested problems using the DonathHoffman bounds given by inequality (12). The bounds generated in Table VIII can be tightened considerably by looking at other diagonal values, $u_{i i}$, satisfying (10). Cullum et al. [6] describe a nondifferentiable mathematical programming approach for selecting $u_{i i}$ values that improve considerably the bounds of the inequality (12).

Rendl and Wolkowicz [18] recently introduced another eigenvector-based scheme for finding bounds on the number of cut edges in a graph with weighted edges. An important feature of their approach is that their bounds are no worse than the Donath-Hoffman bounds given by inequality (12). Preliminary results on small test examples (i.e., up to 100 nodes) reveal that their approach generates bounds that are $25-200 \%$ better than the DonathHoffman bounds. This approach has the added advantage that it avoids the nondifferentiable mathematical programming analysis of Cullum et al. [6] required to compute these bounds. This latter approach is being investigated by the authors.

\section{Conclusions}

In this paper, we have introduced a new method to obtain initial node partitions for use in interchange heuristics where the aim is to minimize the number of nets (wires or signals) cut by the partition. This heuristic relies on a method to approximate a hypergraph or netlist by a graph with weighted edges in order to apply known partitioning results of Barnes [2] based on eigenvectors. The heuristic is very efficient in that the graph approximation of the hypergraph is easily obtained and the most expensive part of the procedure is finding and sorting the eigenvalues and eigenvectors corresponding to the adjacency matrix of the estimating graph.

An efficient implementation of Sanchis's interchange algorithm [20] is used to further reduce the number of cut nets for the $k$-partitions that are initially generated by the new eigenvector model. The outlined approach is tested on several small (300 modules / nets) to large size (12000 modules/nets) netlist partitioning examples. This new approach is compared with the use of at most 30 random starting partitions and the interchange algorithm alone on these test examples.

Test results show that the new approach is comparable to the interchange approach on small examples but generates positions that are $50-100 \%$ better on the larger test cases. The largest test problems, Bio and Industry2, show the importance of getting the best partition possible from using a node interchange approach only once. The running time of the interchange method from one starting partition is becoming expensive. Thus, the method becomes more attractive for solving these practical large cases.

The calculation of the $k$ largest eigenvalues and corresponding eigenvectors for larger netlists by the block Lanczos code is modest [21]. This is accomplished by deleting the nets connected to a large number of modules $(>20$ modules). This has the effect of not introducing largesized cliques in the graph with weighted edges; that is, the approximating graph of the netlist is kept sparse. Future practical codes should attempt to balance denser graph approximations and the running time to find initial partitions. This work is currently in progress.

\section{REFERENCES}

[1] I. Adler, N. Karmarkar, M. G. C. Resende, and G. Veiga, "An implementation of Karmarkar's algorithm for linear programming," Mathematical Programming, vol. 44, pp. 297-335, 1989.

[2] E. R. Barnes, "An algorithm for partitioning the nodes of a graph," SIAM J. Algebraic and Discrete Methods, vol. 3, no. 4, pp. 541$550,1982$. 
[3] J. P. Blanks, "Near-optimal placement using a quadratic objective function,"' in Proc. Design Automat. Conf., 1985.

[4] R. K. Brayton, G. Hactel, K. Mullen, and A. Sangiovanni-Vincentelli, Logic Minimization Algorithms for VLSI Synthesis. Boston Kluwer Academic Publishers, 1984.

[5] A. M. Comeau, "A study of user program optimization in a paging system,"' in Proc. ACM Symp. Operating System Principles (Gatlingburg, TN), Oct. 1967.

[6] J. Cullum, W. E. Donath, and P. Wolfe, "The minimization of certain nondifferentiable sums of eigenvalues of symmetric matrices,' Mathematical Programming Study, vol. 3, pp. 35-55, 1975.

[7] J. Cullum and R. H. Willoughby, Lanczos Algorithms for Large Sym metric Eigenvalues Computation, vols. I and II. Boston: Birkhauser, 1985.

[8] P. J. Denning, "Virtual memory," Comput. Surveys, vol. 2, pp. 153$189,1970$.

[9] W. E. Donath and A. J. Hoffman, "Lower bounds for the partitioning of graphs," IBM J. Res. Develop, vol. 17, no. 5, pp. 420-425, 1973.

[10] C. M. Fiduccia and R. M. Mattheyses, "A linear-time heuristic for improving network partitions," in Proc. 19th Design Automat. Conf. 1982, pp. 175-181.

[11] A. D. Friedman and P. R. Menon, Theory and Design of Switching Circuits. Rockville, MD: Bell Telephone Laboratories and Computer Sciences Press, 1975.

[12] M. R. Garey and D. S. Johnson, Computers and Intractability. San Francisco, CA: Freeman, 1979.

[13] J. R. Gilbert and E. Zmijewski, "A parallel graph partitioning algorithm for a message passing multiprocessor,' Int. J. Parallel Programming, vol. 16 , pp. 427-449, 1987

[14] K. Hall, "An $r$-dimensional quadratic placement algorithm," Management Sci., vol. 17, pp. 219-229, 1970.

[15] N. Karmarkar, "A new polynomial-time algorithm for linear programming," Combinatorica, vol. 4, no. 4, pp. 373-395, 1984

[16] B. W. Kernighan and S. Lin, "An efficient heuristic procedure for partitioning graphs," Bell Syst. Tech. J., vol. 49, no. 2, pp. 291 $307,1970$.

[17] A. Pothen, H. D. Simon, and K. P. Liou, "Partitioning sparse matrices with eigenvectors of graphs," SIAM J. Matrix Analysis and Appl., vol. 11, pp. 430-452, 1990.

[18] F. Rendl and $\mathbf{H}$. Wolkowicz, "A projection technique for partitioning the nodes of a graph," Research Report CORR 90-20, Dept. Combinatorics and Optimization, University of Waterloo, 1990 (submitted to Mathematical Programming).

[19] R. L. Russo, P. H. Oden, and P. K. Wolff, Sr., "A heuristic procedure for the partitioning and mapping of computer logic blocks to modules," IEEE Trans. Comput, vol. C-20, pp 1455-1462, 1972.

[20] L. A. Sanchis, "Multiple-way network partitioning," IEEE Trans. Comput., vol. 38, no. 1, pp. 62-81, 1989.

[21] D. S. Scott, "LASO2 documentation," Computer Science Department, University of Texas at Austin, 1980.

[22] A. J. Vakharia, "Methods of cell formation in group technology: A framework for evaluation," Int. J. Operations Management, vol. 6 no. 3, pp. 257-271, 1986.

[23] A. Vannelli and K. R. Kumar, "A method for finding minimal bottleneck cells for grouping part-machine families," Int. J. Prod. Res. vol. 24 , pp. $387-400,1986$
[24] A. Kusiak, A. Vannelli, and K. R. Kumar, "Clustering analysis: Models and algorithms," Control and Cybernetics, vol. 15, no. 2, pp. $139-154,1986$

[25] A. Vannelli and S. W. Hadley, "A Gomory-Hu cut tree approach for partitioning netlist," IEEE Trans. Circuits Syst., vol. 37, no. 9, pp. 1133-1139, 1990.

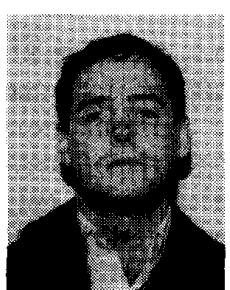

Scott W. Hadley received the B.Math, M.Math and Ph.D. (combinatorics and optimization) degrees from the University of Waterloo, Waterloo, Ontario, Canada, in 1981, 1987, and 1990

$\mathrm{He}$ is presently a Research Mathematician in the Department of Mathematics and Systems Engineering at Koninkliijke/Shell-Laboratorium, Amsterdam, Netherlands. His main research interests lie in the area of practical applications of largescale combinatorial optimization problems.

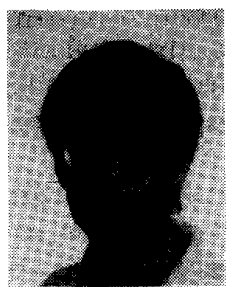

Brian L. Mark received the B.A.Sc. degree in electrical and computer engineering from the University of Waterloo, Waterloo, Ontario, Canada, in 1991 . He is currently undertaking his graduate studies (M.A.Sc. and Ph.D.) in electrical engineering at Princeton University, Princeton, NJ. His main research interests lie in communication theory.

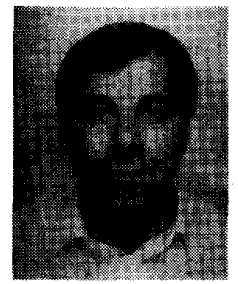

Anthony Vannelli received the $\mathrm{Ph} . \mathrm{D}$. degree in electrical engineering from the University of Waterloo, Waterloo, Ontario, Canada, in 1983. In 1983 and 1984 he was an IBM postdoctoral fellow in the Mathematical Sciences Department at the IBM Thomas J. Watson Research Center, Yorktown Heights, NY.

He joined the Department of Industrial Engineering at the University of Toronto in 1984. In 1987, he joined the Department of Electrical and Computer Engineering at the University of Waterloo. He is currently an Associate Professor and has held a Natural Sciences and Engineering Council of Canada University Research Fellowship since 1984. His research focuses mainly on the development of efficient linear, nonlinear, and combinatorial optimization techniques for solving VLSI circuit layout and design problems. 